\title{
Swoop: A 'Web' Ontology Editing Browser
}

\author{
$\star$ \\ Aditya Kalyanpur ${ }^{1}$, Bijan Parsia ${ }^{2}$, Evren Sirin $^{1}$, \\ Bernardo Cuenca Grau ${ }^{3}$, James Hendler ${ }^{1}$ \\ University of Maryland, MIND Lab, 8400 Baltimore Ave, \\ College Park MD 20742, USA \\ \{aditya, evren, hendler $\}^{1} @$ cs.umd.edu, bparsia@isr.umd.edu ${ }^{2}$, \\ bernardo@mindlab.umd.edu ${ }^{3}$
}

\begin{abstract}
In this paper, we describe Swoop, a hypermedia inspired Ontology Browser and Editor based on OWL, the recently standardized Web-oriented ontology language. After discussing the design rationale and architecture of Swoop, we focus mainly on its features, using illustrative examples to highlight its use. We demonstrate that with its web-metaphor, adherence to OWL recommendations and key unique features such as Collaborative Annotation using Annotea, Swoop acts as a useful and efficient web ontology development tool. We conclude with a list of future plans for Swoop, that should further increase its overall appeal and accessibility.
\end{abstract}

Key words: OWL, Ontology Engineering, Systems

\section{Introduction: Design Rationale and Goals}

Swoop is built primarily as a Web Ontology Browser and Editor, i.e., it is tailored specifically for OWL [1] ontologies. Thus, it takes the standard Web browser as the UI paradigm, believing that URIs are central to the understanding and construction of OWL Ontologies. The familiar look and feel of a browser emphasized by the address bar and history buttons, navigation side bar, bookmarks, hypertextual navigation etc are all supported for web ontologies, corresponding with the mental model people have of URI-based web tools based on their current Web browsers (see Fig. 1).

ॠ Please note that this paper is intended to be a systems and not a research paper 


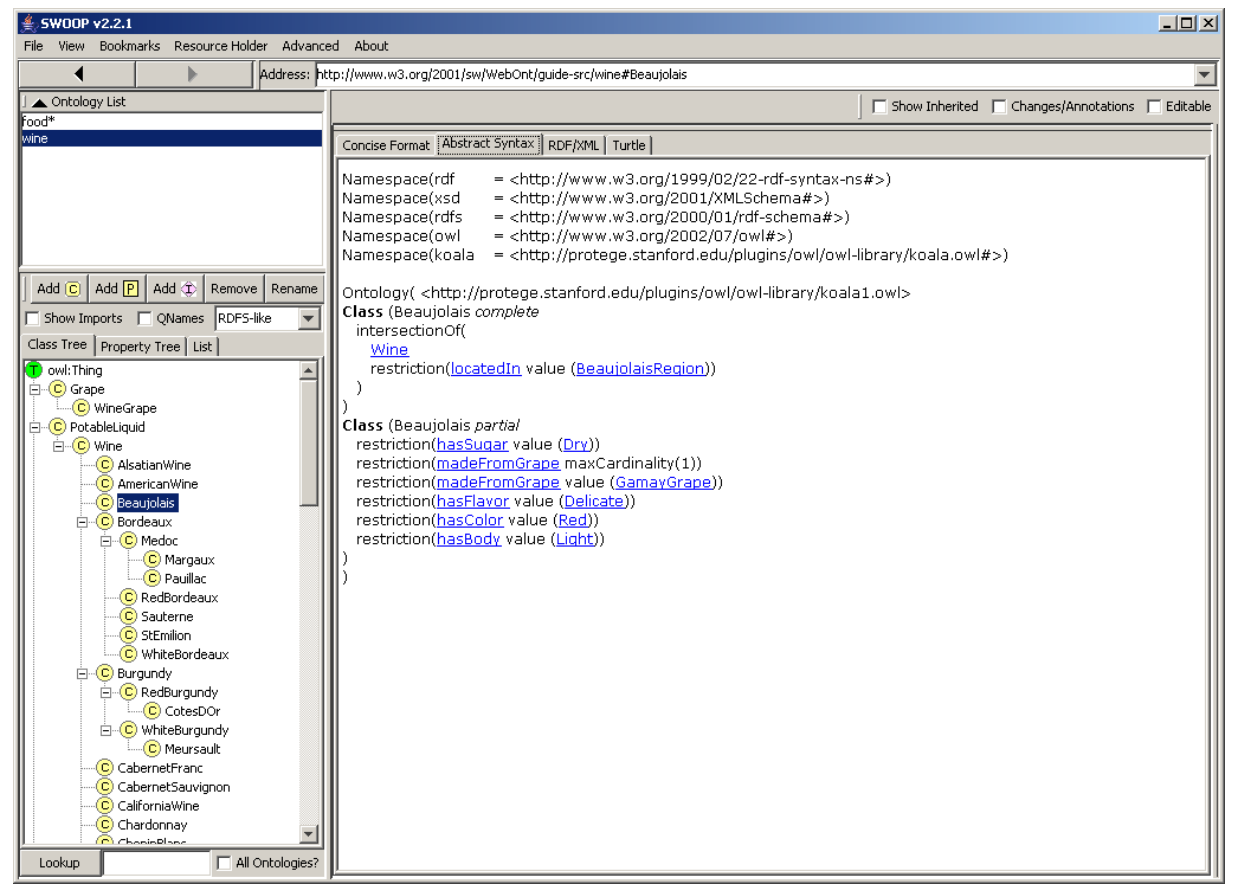

Fig. 1. Swoop: A Web Ontology Browser. The layout resembles a familiar frame-based website viewed through a Web browser. A navigation sidebar on the left contains the multiple ontology list and class/property hierarchies for each ontology, while the center pane contains the various ontology/entity renderers for displaying the core content. The history buttons (back, next) and address bar take their familiar position at the top of the frame.

All design decisions are in keeping with the OWL nature and specifications. Thus, multiple ontologies are supported easily, various OWL presentation syntax are used to render ontologies, OWL reasoners can be integrated for consistency checking, and open-world semantics are assumed while editing. To give an example of the latter aspect, some ontology editors make the assumption that an owl:FunctionalProperty can only allow one value in all cases thus providing a single input component. However, this is not true in the case of OWL ObjectProperties where multiple values (individuals) can exist for the functional property, allowing the reasoner to infer equivalence between the values.

A key point in our work is that the hypermedia basis of the UI is exposed in virtually every aspect of ontology engineering - easy navigation of OWL entities, comparing and editing related entities, search and cross referencing, multimedia support for annotation, etc. - thus allowing ontology developers to think of OWL as just another Web format, and thereby take advantage of its Web-based features.

A diverse array of ontology related tasks can be performed in Swoop ranging from collaborative annotation and data markup to ontology refactoring and debugging. This makes Swoop accessible to both, novice users interested in 
casual ontology building and use [2], and expert users interested in robust ontology modeling and analysis.

\section{Related Work}

There exist numerous ontology development toolkits (many of which have OWL support), that provide an integrated environment to build and edit ontologies, check for errors and inconsistencies (using a reasoner), browse multiple ontologies, and share and reuse existing data by establishing mappings among different ontological entities.

However, we differentiate Swoop on several aspects. First, to our knowledge, Swoop is the only ontology editor currently available that is catered wholly towards OWL from the ground up and its design rationale reflects this. Second, it contrasts with other ontology editors such as Protégé [3], OilED [4], and OntoEdit [5] which either are, or were influenced by traditional KR development tools and applications, and do not reflect "Webiness" in their UI design. In particular, they do not fully support the use of hypertext to drive the exploration and editing of ontologies.

Also, as mentioned in [6], there are several examples of current website-based ontology tools(e.g. Ontosaurus [7], WebODE [8]), pOWL ${ }^{1}$ ). However, we have found that using a standard web-based server-client architecture for ontology engineering suffers from being slow (esp. for large ontologies, and depending on network traffic), and cumbersome for maintaining consistency while editing (eg. trapping input errors, changing/deleting objects but reloading from browser cache etc), an argument also supported in [9]. In addition, such tools can be difficult to extend to new functionalities via plug-in architectures (such as the one used in Swoop). For these reasons, Swoop is developed as a separate Java application that attempts to provide the look and feel of a browser-based application, but with its specialized architecture designed to optimize OWL browsing and to be extensible via a plug-in architecture.

\section{Swoop Architecture}

Swoop is based on the Model-View-Controller (MVC [10]) paradigm. The SwoopModel component stores all ontology-centric information pertaining to the Swoop Workspace (currently loaded ontologies, change-logs, checkpoints) and defines key parameters used by the Swoop UI objects (such as selected

$\overline{1 \text { pOWL }}$ - http://powl.sourceforge.net 


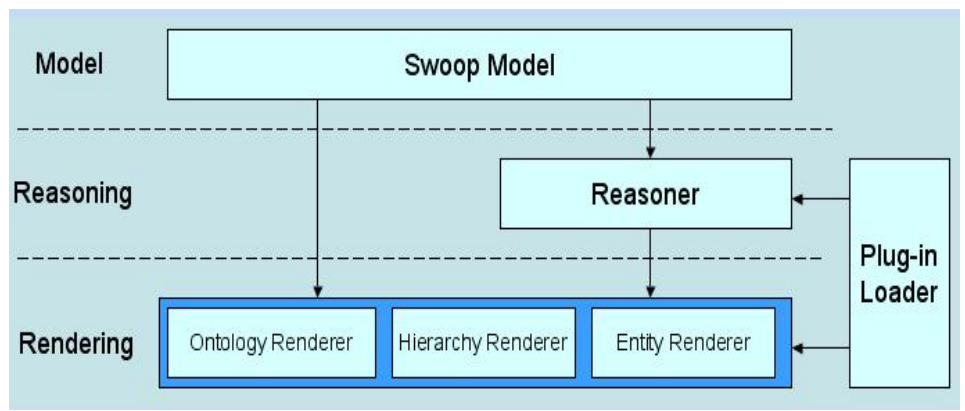

Fig. 2. Plugin-based Swoop Architecture

OWL entity, view settings for imports, QNames etc). Additionally, a SwoopModelListener class is used to reflect changes in the UI based on changes in the SwoopModel (using a suitably defined event-notification scheme). Control is handled through a plugin based system, which loads new Renderers and Reasoners dynamically. The obvious advantage of a plugin framework is to ensure modularity of the code, and encourage external developers to contribute to the Swoop project easily. Finally, we note that the entire Swoop code is written in Java, maintained in a subversion repository and makes use of numerous third party libraries, the most prominent being the WonderWeb OWL API [11] which is used as the underlying OWL representation model.

\section{Swoop Features}

In this section, we describe the features of Swoop that are in keeping with its design rationale and goals mentioned earlier. In particular, we focus on the unique aspects of 'Web' Ontologies and the corresponding tool support needed to develop each aspect effectively. Note that all features mentioned in this paper are with reference to the v2.2 release of Swoop, which can be obtained at the Swoop website: http://www.mindswap.org/2004/SWOOP.

\subsection{Ontologies based on the Web Architecture}

The idea behind Web ontology development is different from traditional and more controlled ontology engineering approaches which rely on high investment, relatively large, heavily engineered, mostly monolithic ontologies. For OWL ontologies, which are based on the Web architecture (characterized as being open, distributed and scalable), the emphasis is more on utilizing this freeform nature of the Web to develop and share (preferably smaller) ontology models in a relatively ad hoc manner, allowing ontological data to be reused easily, either by linking models (using the numerous mapping properties available in OWL) or merging them (using the owl: imports command). Thus, it 
becomes essential for a Web ontology development tool to scale to multiple ontologies easily, and to allow tasks such as creation, browsing, editing, search, reuse, linking, merge/split of OWL ontology models in the context of multiple large distributed ontologies.

In order to attain this key requirement, Swoop ensures that users are free to load multiple OWL ontologies in any manner they prefer. The easiest and most direct way to load an OWL Ontology is by entering its physical URL (Web or local file address) in the address bar. This action not only pulls in the requested ontology, but also loads any imported ontologies (defined using owl:imports) into Swoop automatically. The bookmarks feature can be used to store, categorize and reload ontologies directly (as is the case in standard web browsers). Finally, depending on user preference, an ontology can also be brought into Swoop rather seamlessly during browsing/editing, e.g., attempting to view or refer to an externally referenced entity while in a particular ontology can load the external ontology automatically.

There are certain characteristics of OWL ontologies which are presented to the user when a new ontology is brought into Swoop. The Ontology Renderer plugins in Swoop accomplish this, and display statistics such as (see Fig. 3):

- the logical constructs used in the ontology model which determine the OWL species level the ontology belongs to, i.e., OWL Lite, DL or Full

- the Description Logic (DL) expressivity of the ontology - a key factor in determining complexity of reasoning

- number of classes, properties, individuals etc. (we intend to extend the granularity to axioms, e.g., no. of disjoint axioms, no. of nominals used etc.)

- annotations on the ontology object itself (including owl:imports)

\subsubsection{Editing Web Ontologies}

Consider a scenario in which a user is building an ontology for the University of Maryland (UMD) for describing its administrative hierarchy (with concepts such as Department, Faculty, Staff, Student etc). This user can make use of existing concepts in well-known upper-level ontologies such as FOAF or Cyc (for generic concepts such as Person), or in similar ontologies created for other universities (such as Stanford or CMU). Another user interested in building a finer-grained ontology than the one above, say for describing his/her research group at UMD, can now use the UMD ontology to refer to or define certain concepts. In this manner, the open development cycle of create-link-share web ontologies ensures that a large amount of interrelated semantic content is available in ontologies. Moreover, it satisfies one of the fundamental necessities of effective ontology development for the Semantic Web, that of semantic interoperability - instance data written in one ontology 


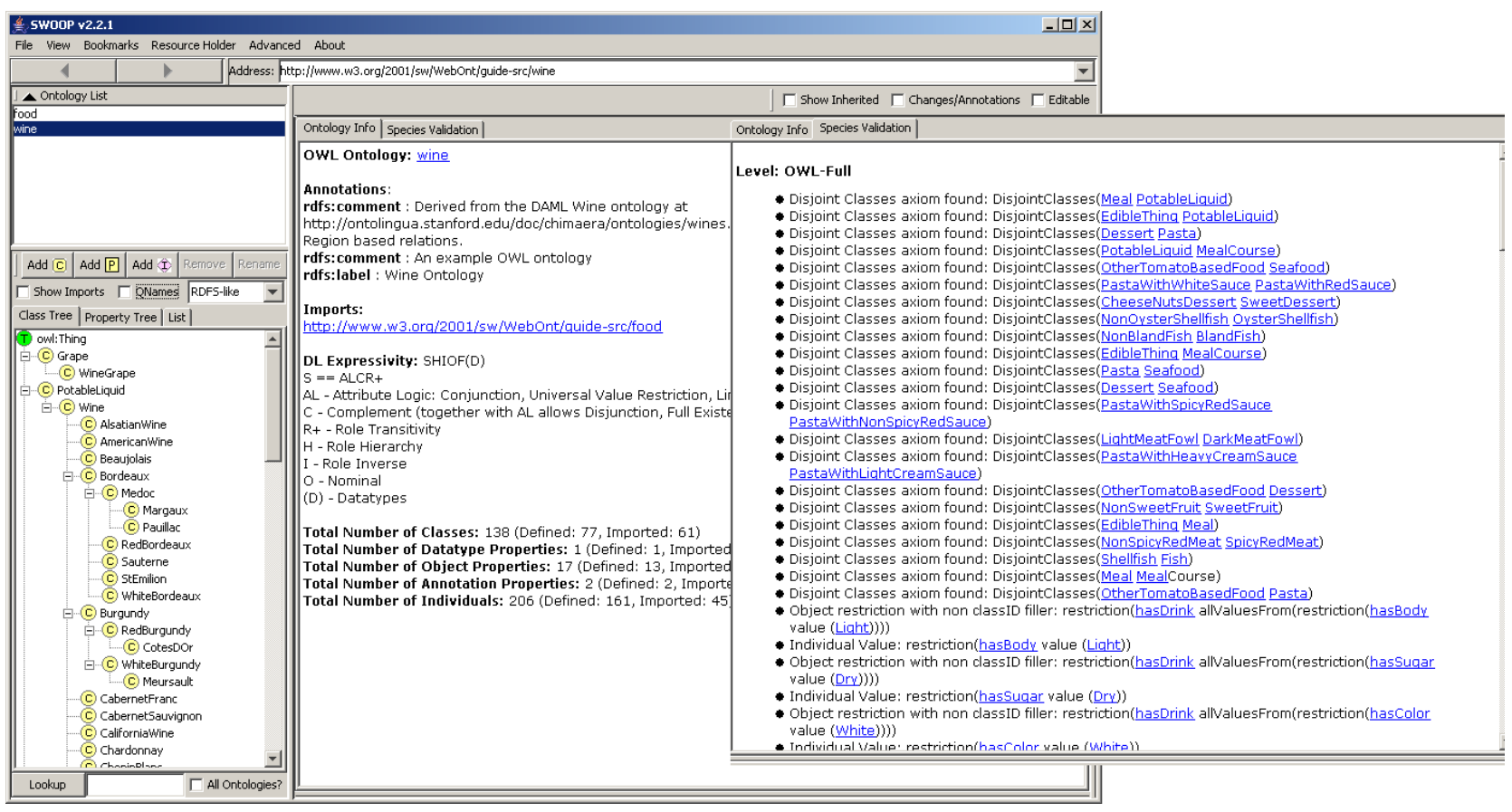

Fig. 3. Swoop Ontology Renderers: display statistical information about the ontology, annotations, DL expressivity and OWL species level.

can be transformed into data for another ontology provided the necessary links exist.

In keeping with the above scenario, Swoop allows users to freely link (map between) entities in different ontologies using a single common interface, which lists each ontology loaded in Swoop along with its corresponding entity list (see Fig: 4).

This seemingly simple feature underlines the true significance and power of Web ontologies as described above, i.e., the ease in which ontological data can be reused creating a semantic mesh of interlinked ontologies such that, as the mesh grows larger and more interconnected, it becomes easier and more useful for other web ontology developers/users to build and utilize specific ontology models on the fly.

It is important to consider the scenario in which a user edits an external ontology present at a URL under the control of a third party. In this case, though the ontology could be considered as read-only, Swoop allows the ontology to be modified and a local version of the ontology to be maintained separately (since it cannot be exported to the URL). However, now, its new physical location is used for reference in an owl: import axiom that specifies importing the external ontology. In general, Swoop tracks import-links across the ontologies in its environment, and when the physical location of an ontology is changed, the corresponding import links are updated as well. 


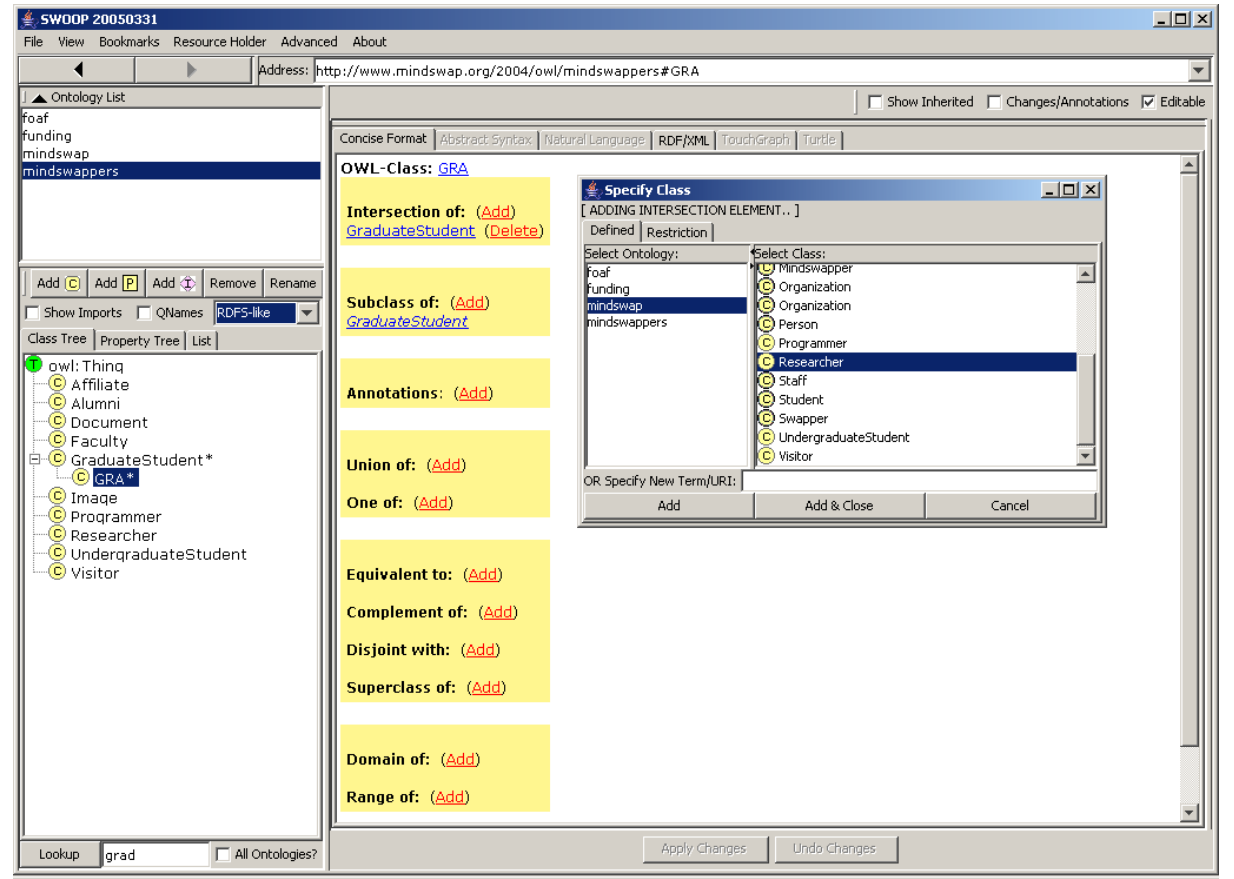

Fig. 4. Editing in Swoop: Clicking the 'Add' hyperlink next to an assertion heading (e.g., Intersection of ) pops up a window to specify corresponding new information (e.g., the new intersection class). In this case, the user is specifying a class Researcher from an external ontology mindswap as an intersection element

However, there are additional caveats to be considered while editing in a multiple ontology setting as described above. For starters, it is essential to provide a search feature to help users find related ontological information. Having found such information, it then becomes critical to compare and analyze this information in order to determine which parts, if any, are useful (verifying relevance, accuracy etc). Finally, the user needs a flexible reuse scheme that supports either borrowing the entire external ontology model if desired, or a subset of it which is relevant, allowing suitable modifications if any. We deal with each of these three caveats in detail as reflected in Swoop.

Search in Swoop essentially performs a lookup for entities (classes / properties/ individuals) across single or multiple ontologies, among those that have been loaded. The results are obtained as a set of hyperlinks (in keeping with the hypermedia-based UI) allowing the user to browse the search results easily (see Fig. 5).

During an extensive search/browsing process, the user may need to set aside and revisit interesting search results. In Swoop we have a provision to store and compare OWL entities via a Resource Holder panel (see Fig 6). Items can be added to this panel at any time and they remain static there until the user decides to remove or replace them at a later stage. This common placeholder acts as an excellent platform for performing interesting engineering tasks such 


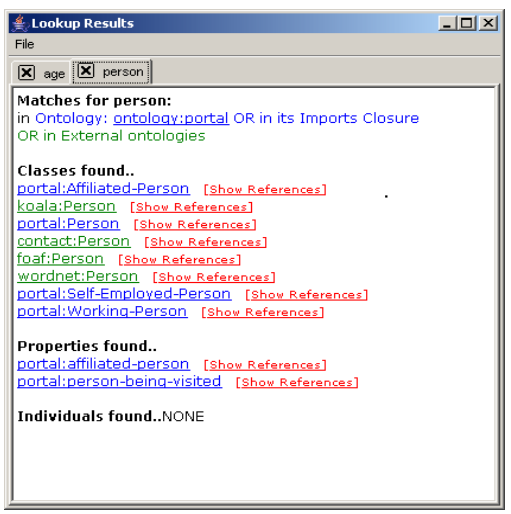

Fig. 5. Ontology Search in Swoop for the keyword 'Person'

as comparing differences in definitions of a set of entities; determining semantic mappings between a specific pair of entities or simply storing entities for reusing in another ontology.

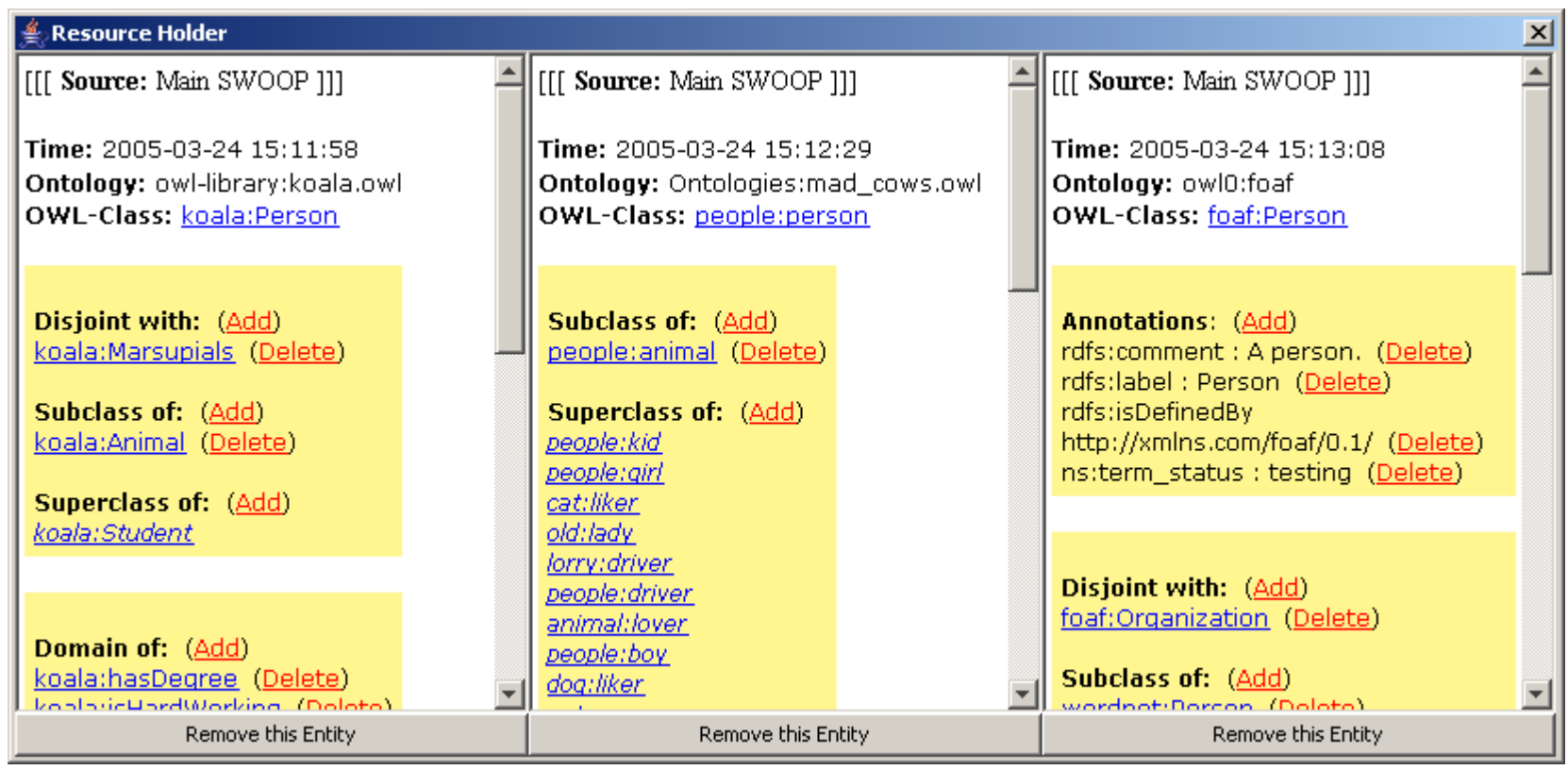

Fig. 6. Resource Holder: used to compare 3 different definitions of the OWL Class Person as specified in 3 different ontologies

\subsubsection{Reusing OWL Data: The Partial Imports Problem}

Having dealt with the important aspects of search and comparison of related data in a multiple ontology setting, we are now left with the critical task of reusing data efficiently. Note that all the constructs in RDFS/OWL (e.g. owl: equivalentTo, rdfs:subClass0f) can be used to relate entities from different ontologies. However, the actual semantics of the linked entities depends on whether the corresponding ontologies import one another, e.g., if class $C_{A}$ (in ontology $A$ ) is defined as equivalentTo class $C_{B}$ (in ontology 
$B$ ), the semantics of $C_{A}$ differ depending on whether $A$ imports $B$ or not. Thus, effectively, there are two ways in which users can reuse external ontological data, i.e., either by simply linking to the external entity, or by linking to the entity and importing the entire external ontology (using owl : imports). Already, one of the key drawbacks of the above reuse scheme has become clear - either users are forced to underspecify the semantics of their model by linking to but not importing the external ontology (using the former approach), or they are forced to overspecify their model by importing the entire semantics, i.e. all axioms, of the imported ontology (using the latter approach). Thus, there is no easy, direct solution for partial imports in OWL. Various workarounds exist such as a brute-force syntactic scheme to copy/paste relevant parts (axioms) of the external ontology, or a more elegant solution that involves partitioning the external ontology while preserving its semantics and then reusing (importing) only the specific partition as desired.

We have explored the latter solution for partitioning OWL Ontologies by transforming them into an E-connection. Describing the theory and significance of E-connections, their use in the context of OWL Ontologies and details of the partitioning algorithm are beyond the scope of this paper. For more on that visit http://www.mindswap.org/2004/multipleOnt. Here we briefly describe the process: an ontology dealing with numerous inter-related domains is transformed into an E-connection which consists of sub-ontologies (partitions) that each deal with a distinct domain. An obvious advantage of the above modularity is that a user interested in reusing a concept from a particular domain, can import only the corresponding partition instead of the entire original ontology.

\subsection{Adhering to OWL Specifications: Presentation and Reasoning}

Currently, various presentation syntax exist for rendering OWL ontologies such as RDF/XML [12], OWL Abstract Syntax [13], and Turtle [14]. It is important to support these different syntax while designing an open, Semantic Web ontology engineering environment. One reason for this is that people tend to have strong biases toward different notations and simply prefer to work in one or another. A second is that some other tool might only consume one particular syntax (with the RDF/XML syntax being the most typical), but that syntax might not be an easy or natural one for a particular user. A third is that it is important to support the "view source" effect, allowing cut and paste reuse into different tools including text editors, markup tools, or other semantic web tools. For these reasons, Swoop has default plugins for all three presentation syntax mentioned above. Users are free to browse and 
edit $^{2}$ ontological data, either at the level of a single entity (inline) or at the level of the entire ontology as a whole, in any syntax as desired, switching between syntax on a single click.

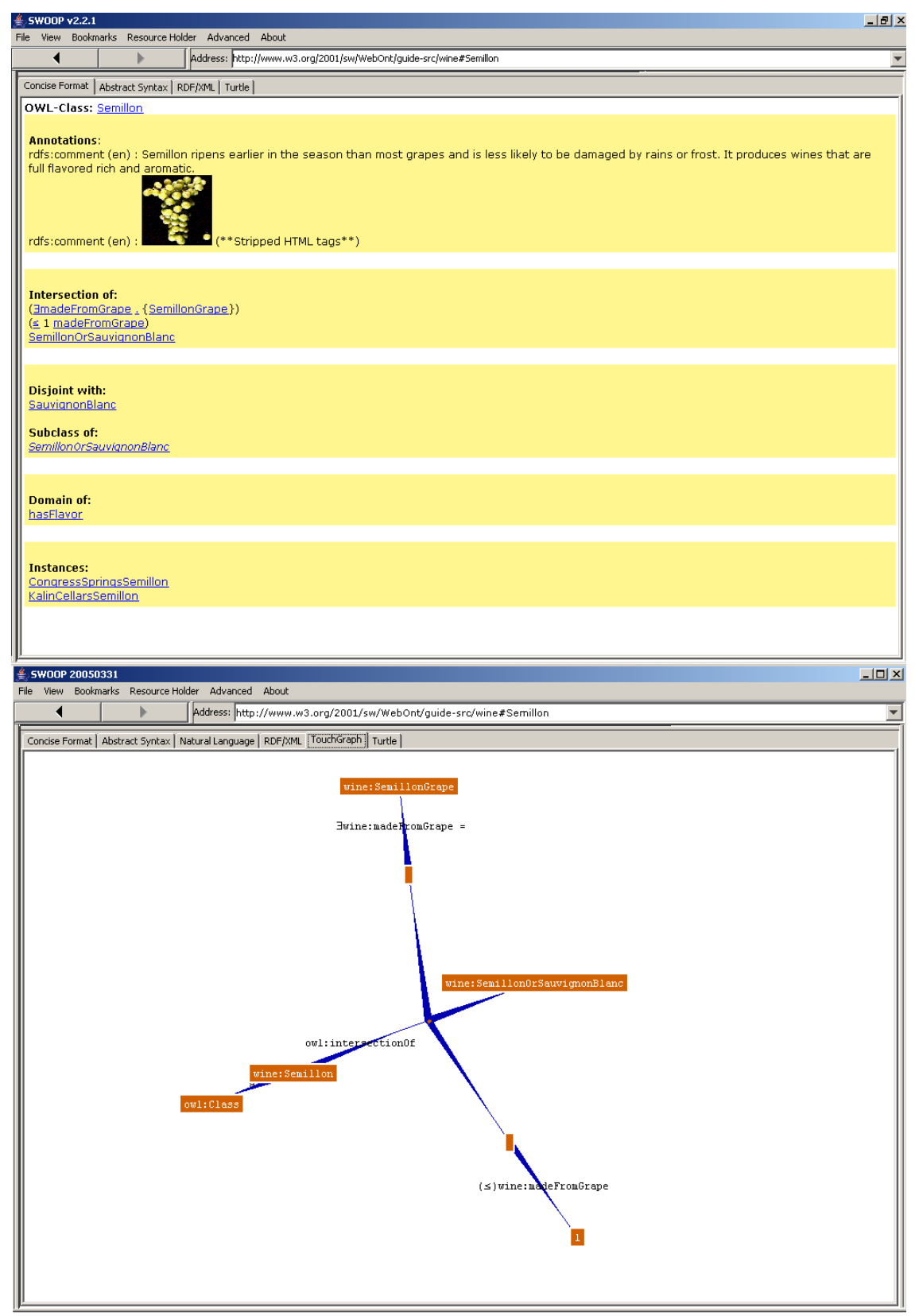

Fig. 7. Top: Concise Format Entity Renderer: displays all ontological information about the OWL Class Semillon as a single Web document Bottom: Graph Visualization plugin: displays a conceptual graph with nodes as classes and edges as properties (restrictions and collections are concisely represented)

In addition to the default OWL presentation syntax, we are working on three

$\overline{2}$ Note: Swoop v2.2 has support for inline editing in RDF/XML, the subsequent release will allow for inline editing in the other two syntax as well 
additional renderers to help users visualize and understand OWL ontologies better (two of which are shown in Fig. 7). These include a Concise Format entity renderer, where the idea is to generate a "Web document" that displays all information related to a particular OWL entity concisely in a single pane; a Natural Language entity renderer that provides concise, accurate NL paraphrases for OWL Concepts based on a variety of NLP techniques [15]; and an OWL Graph visualization renderer based on TouchGraph that displays concise conceptual graphs of the ontology model. Each of these renderers provide a different view of the model, allowing users to understand logical definitions and relationships better.

\subsubsection{Reasoning in $O W L$}

Having covered the presentation aspects of OWL ontologies, we now focus on the reasoning support in Swoop. Note that OWL-DL is primarily based on description logic, with open-world semantics and a non unique name assumption (UNA). Swoop strictly maintains the latter two aspects during editing, e.g., it does not try to 'interfere' with creating the KB (i.e., prevent the creation of inconsistencies) by making any additional alterations or assumptions, and accurately reflects the users' actions based on open world semantics. As for the DL reasoning, Swoop allows for special-purpose reasoner plugins that provide standard reasoner services such as satisfiability (of a single class as well as consistency of the ontology), subsumption (between classes and between properties), and realization (types of an instance). Additionally, reasoners can support the optional explanations feature, which is used for sophisticated ontology debugging as explained later.

Swoop contains two additional reasoners (besides the basic Reasoner that simply uses the asserted structure of the ontology): RDFS-like and Pellet [16]. While the former is a lightweight reasoner based on RDFS semantics, the latter, Pellet, is a powerful description logic tableaux reasoner. Pellet has a number of advantages: It natively supports OWL, including a repairable subset of OWL Full; it has extensive support for XML Schema datatypes; it has ABox (a.k.a., instance) support; it covers the broadest range of OWL DL of any reasoner that we know, including both $\mathrm{SHIN}(\mathrm{D}), \mathrm{SHON}(\mathrm{D}), \mathrm{SHIO}(\mathrm{D})$, and various subsets of their union, $\operatorname{SHOIN(D)~(a.k.a.,~OWL~DL);~it~is~open~}$ source and in active, public development. The last is very important for certain debugging strategies which require access to the internals of the reasoner as noted later.

The above reasoners provide a tradeoff between speed and quality of inference results, e.g., the RDFS-like reasoner, while much faster than Pellet in execution, is unsound (results maybe inaccurate if the ontology is inconsistent) and incomplete (does not list all possible inferences). Yet, in most cases, it 
provides interesting and useful results for ontology authors, and moreover, the reasoners can be used in conjunction to analyze the ontology quickly while editing it. Fig. 8 illustrates the use and functionality of the various reasoners in Swoop, by highlighting the difference in the results provided for the same class definition in a given ontology.

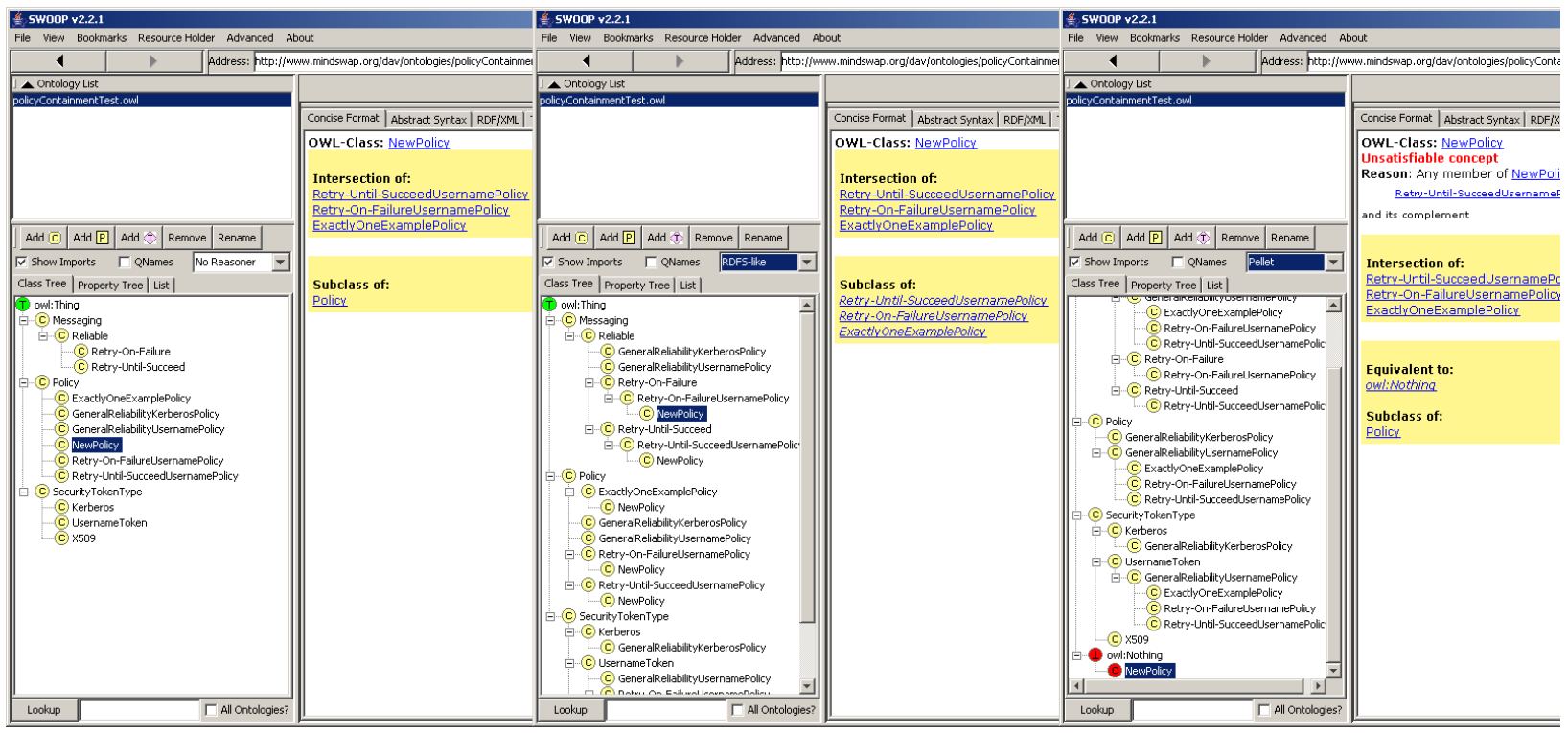

Fig. 8. Using the Swoop Reasoners: Note the change in position of the class NewPolicy in the subsumption graph when the reasoner selection changes. Initially, it is known to be a subclass of Policy in the asserted (No Reasoner) mode; then inferred to be a subclass of three distinct classes in the RDFS mode, and finally found to be unsatisfiable in Pellet mode.

\subsubsection{Ontology Debugging and Repair}

DL reasoners such as Pellet, RACER [17], FACT [18] etc can be used to detect inconsistencies in definitions of concepts (a.k.a. unsatisfiable concepts). However, typically reasoners only report that a class is unsatisfiable, not why. Moreover, they do not report on inter-dependencies (if any) of the unsatisfiable classes, i.e., if a class directly depends on another for its unsatisfiability (e.g., by an existential property restriction on an unsatisfiable class). We argue that both forms of explanation are essential for the purpose of debugging ontologies; while the former can be used to understand and rectify problematic axioms / class expressions, the latter can help prune out dependency bugs and let the modeler focus on the root (source) of the problem alone.

We distinguish two families of reasoner-based techniques for supporting diagnosis of the form described above: glass box and black box techniques. In glass box techniques, information from the internals of the reasoner is extracted and presented to the user (typically used to pinpoint the type of clash/contradiction and axioms leading to the clash). In black box techniques, 
the reasoner is used as an oracle for a certain set of questions e.g., the standard description logic inferences (subsumption, satisfiability, etc.) and the asserted structure of the ontology is used to help isolate the source of the problems (can be used to find dependencies between unsatisfiable classes). Presenting the details of both approaches is beyond the scope of this paper, for more see [19].

For now, we note that both forms of ontology debugging in Swoop is exposed through reasoners which support the optional explanations service (Pellet by default does this), and describe a simple example to illustrate the use of debugging: Consider the case of the unsatisfiable class Koala depicted in Figure 9. Swoop displays a one line quasi-Natural Language description explaining the cause of the clash in the class definition, followed by a list of the relevant set of axioms from the ontology responsible for the clash. This provides a direct pointer to the problematic part of the ontology as making this class satisfiable involves removing or fixing any one of the axioms displayed.

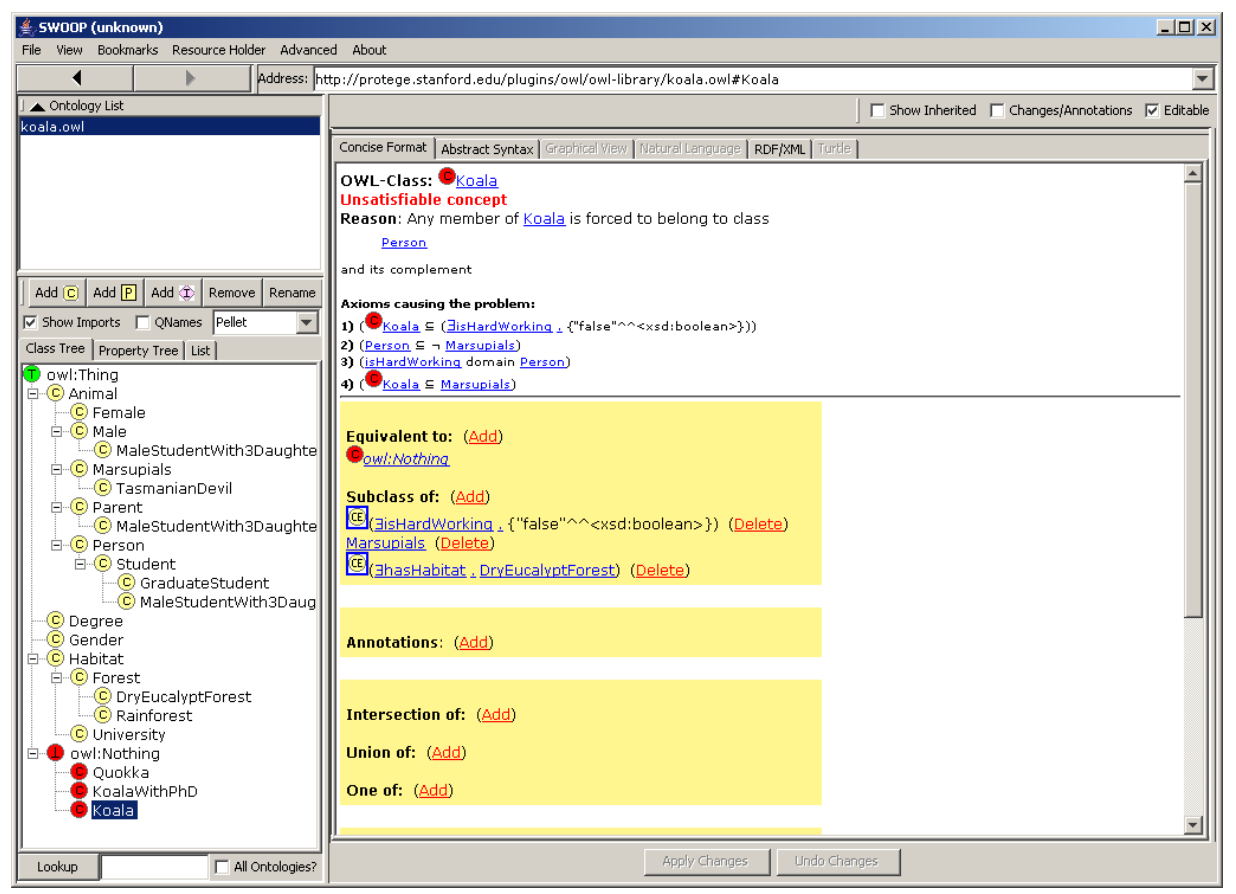

Fig. 9. The set of axioms that support the inconsistency of Koala concept is displayed in debug mode.

\subsection{Extending the Swoop Framework for Collaborative, Distributed Web On- tology Evolution}

Till now we have seen how Swoop makes use of the Web-based nature of OWL Ontologies and remains true to the OWL specifications while providing for an open and scalable hypermedia-inspired ontology development environment. 
Now, we extend the distributed nature of Web-based ontologies to support collaborative ontology evolution in Swoop. Our goal is to allow a group of ontology authors, possibly working in a remote environment (i.e., on separate machines), to develop and evolve a set of OWL ontologies together. The set of tasks we would like to support include:

- Easy Exchange of Ontological Data at different granularity levels such as a set of ontologies/entities, a single ontology/entity or a change set.

- Collaborative Annotation for exchanging and discussing ideas during all stages of the ontology development cycle

- Version Control for efficiently maintaining the ontologies over time by logging all changes and regulating its progress

- Unit Testing to ensure that the ontology satisfies certain predetermined criteria and goals

An underlying objective of the above tasks is to make full use of existing standards for representing, annotating and versioning ontological data, e.g., using versioning constructs of OWL such as owl:versionInfo owl:priorVersion, owl: backwardCompatibility and owl:deprecated.. where necessary.

We note that work towards this feature is still in progress. For now, we describe the middle two components (listed above) that are fairly well developed and usable in their own right.

For collaborative annotation in Swoop, we use the Annotea framework [20], which takes the idea of separating annotations about ontologies from the core ontologies themselves and provides both a specific RDF based, extensible annotation vocabulary, and a protocol for publishing and finding out-of-band annotations (annotations that do not live inside the document being annotated).

Annotea support in Swoop is provided via a simple plug in whose implementation is based on the standard W3C Annotea protocols [21] and uses the default Annotea RDF schema to specify annotations (see Fig. 10). Any public Annotea Server can then be used to publish and distribute the annotations created in Swoop. The default annotation types (comment, advice, example, etc) seem an adequate base for human oriented ontology annotations. One extension we have begun experimenting with is "Prototypicallllustration", that is, a photo or drawing that represents a typical or canonical instance of the class.

We have extended the Annotea Schema with the addition of an OWL ontology for a new class of annotations - ontology changes (similar to [22]). The "Change" annotation defined by the Annotea projected was designed to indicate a proposed change to the annotated document, with the proposal described in HTML-marked-up natural language. In our extended ontology, 


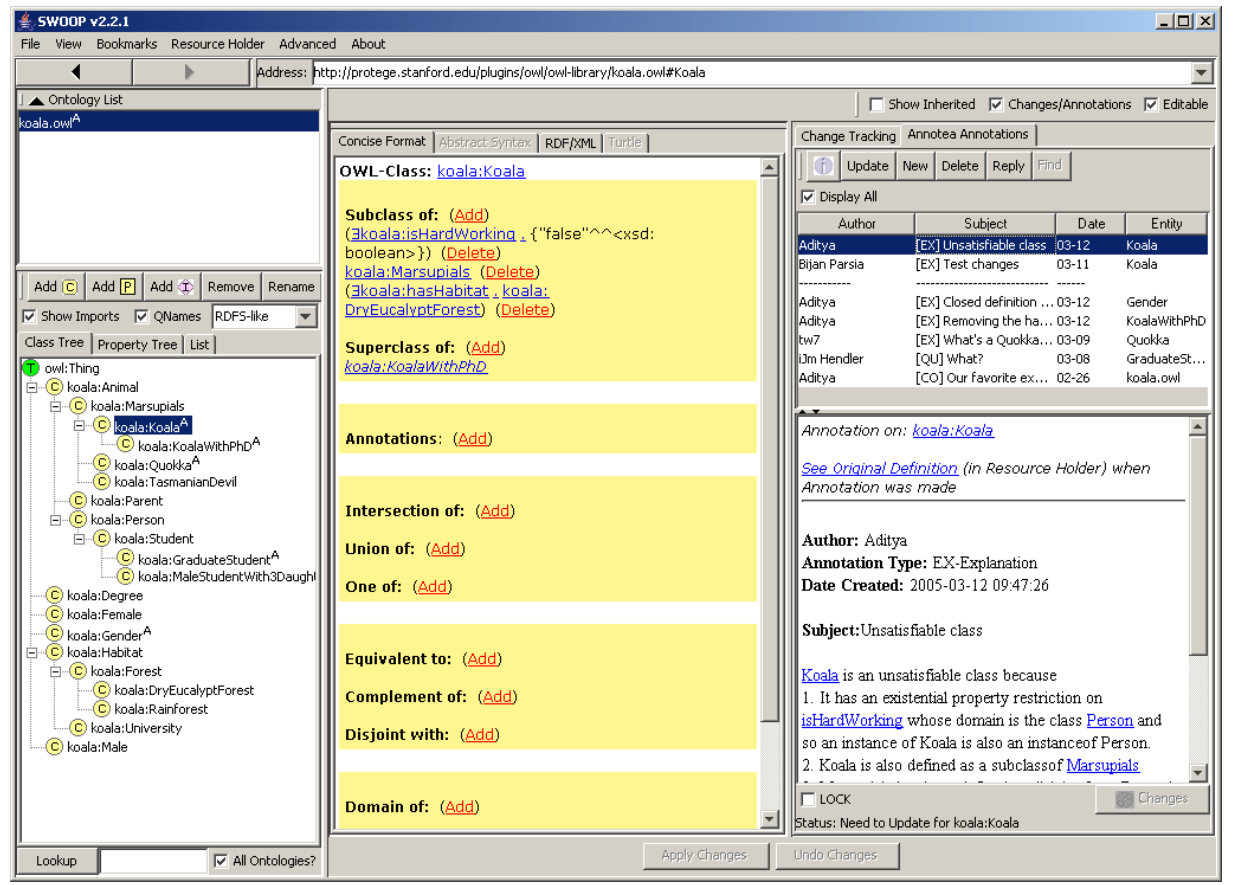

Fig. 10. Annotea in Swoop: Collaborative Annotation and Discussion of OWL Entities using the Annotea Client.

change individuals correspond to specific changes made in Swoop during editing. Note that Swoop uses the OWL API to model ontologies and their associated entities, benefiting from its extensive and clean support for changes. The OWL API separates the representation of changes from the application of changes. Each possible change type has a corresponding Java class in the API, which is subsequently applied to the ontology (essentially, the Command design pattern). These classes allow for the rich representation of changes, including metadata about the changes.

The Swoop change annotations can be published and retrieved by Annotea servers, or any other annotation distribution mechanism. The retrieved annotations can then be browsed, filtered, endorsed, recommended, and selectively accepted. A similar collaborative framework based on an interactive dialogue was implemented in a more local (tool-specific) context in the WebOnto system [9]. However, we decided to exchange annotations using the Annotea protocol to make the collaboration less tool-specific (any Annotea client can be used to discuss ontology annotations), and to allow users to arbitrarily extend the Annotea schema the way we have for ontology-change sets. These change sets also make it possible to define "virtual versions" of an ontology, by specifying a base ontology and a set of changes to apply to it (a feature present since the Swoop v2.2 release).

Note that in certain cases, changes may not be applicable to the ontology, if the change operation refers to an entity that is not present (defined) in the 
ontology. In such cases, a warning message is reported to the user describing the reason for the change conflict.

Finally, regarding version control, Swoop supports ad hoc undo/redo of changes (with logging) coupled with the ability to checkpoint and archive different ontology versions. Each change set or checkpoint can be saved at three different granularity levels - entity, ontology, workspace, which basically specify its scope. While the change logs can be used to explicitly track the evolution of an ontology ${ }^{3}$, checkpoints allows the user to switch between versions directly exploring different modeling alternatives. Swoop also has the option to save checkpoints automatically, i.e., during specific tasks such as loading a new ontology, applying changes, removing or renaming an entity etc. Note that each time a checkpoint is saved, a snapshot of the entity definition is cached as well, and can be used to preview a checkpoint before reverting back to it.

The ontology evolution framework in Swoop is modeled on Smalltalks notion of change records and sets and is not as advanced as that in KAON [23], which has a special-purpose API for systematically controlling composite changes, applying change strategies and ensuring consistency during modification. In the future, we plan to learn from and improve the flexibility of the evolution scheme based on systems such as KAON.

\section{$5 \quad$ Immediate Future Plans}

Currently, search is implemented as a straightforward (sub)string matching algorithm that looks in entity name declarations, and works very fast since all indexing is native to Swoop. We are working on extending this naive search algorithm (and the associated indexing scheme) to handle more complex regular expressions and to look inside entity annotations (such as rdfs: comment). We are also investigating class expression search to support anonymous classes (b-nodes) in queries such as 'Find all classes which contain $\exists p . C$ ', and more advanced structural search to support queries such as 'Find all classes $C$, such that property $p_{1}, p_{2}, p_{3}$ have $C$ as its domain'. Using these various search routines, users can customize their queries to find highly specific or generic matches based on their interests.

We also plan on developing an Advanced Resource Holder that would feature automatic dynamic tracking for selected entities, color coding diffs between different entity definitions, and providing support for the editing of mapping terms, such as "owl:equivalentTo" between terms in different re-

$\overline{3}$ Change logs can also be serialized in RDF/XML and exchanged among users 
source panes. Additionally, the Swoop v2.2 release has preliminary support for sound and complete conjunctive ABox queries written in RDQL [24]. We plan to extend this feature to include hybrid TBox/ABox queries as well as non-standard structural queries. Finally, since the OWL-API has support for SWRL [25], we plan to develop an intuitive UI and integrate a Rule Engine into Swoop for writing and processing rules.

\section{Conclusion}

In this paper, we describe Swoop, a hypermedia inspired Ontology Browser and Editor based on OWL, the first standardized Web-oriented ontology language. In designing Swoop, we take the familiar Web browser as our User Interface (UI) paradigm, focusing on a simple and intuitive ontology development interface. The primary goal has been to design a tool based on the successful architecture of the Web itself, i.e., its open (ad hoc local and remote multiple ontology support, various OWL Presentation syntax, extensible plugin architecture), scalable (e.g., ontologies as large as NCI [26] with over 27000 classes can be loaded in Swoop is under 2 minutes), and distributed (e.g., collaborative annotation support via Annotea). By doing the above, and remaining true to the OWL specifications, we allow the Swoop user to take advantage of the Web-based features of OWL.

Our secondary goal has been on overcoming the drawbacks, if any, of traditional ontology development tasks as applied to OWL. For instance, we have presented a partitioning approach for OWL ontologies to solve the partial owl:imports problem while facilitating ontology reuse. We have also presented work on guiding the user through the process of ontology debugging and repair (using explanations), where standard DL Reasoners do not provide additional help.

Finally, we are working on extending Swoop to further aid various forms of Web ontology development such as distributed, collaborative ontology evolution, the pieces of which already exist as separate Swoop components.

In this manner, with the strong platform Swoop provides for Web ontology development and its easy extensibility, Swoop is both, accessible and useful, for OWL developers and users. 


\section{Acknowledgments}

The authors would wish to thank Ron Alford, Taowei David Wang, Vladimir Kolovski, Daniel Hewlett, and Michael Grove for their contribution in developing Swoop and the associated API's/plugins.

This work was completed with funding from Fujitsu Laboratories of America - College Park, Lockheed Martin Advanced Technology Laboratory, NTT Corp., Kevric Corp., SAIC, National Science Foundation, National GeospatialIntelligence Agency, DARPA, US Army Research Laboratory, NIST, and other DoD sources.

\section{References}

[1] M. Dean, G. Schreiber, OWL Web Ontology Language Reference W3C Recommendation. http://www.w3.org/tr/owl-ref/.

[2] A. Kalyanpur, N. Hashmi, J. Golbeck, B. Parsia, Lifecycle of a casual web ontology development process, in: Proceedings of the WWW2004 Workshop on Application Design, Development and Implementation Issues in the Semantic Web, May 18, 2004, 2004.

URL http://www.mindswap.org/ aditkal/WWW04 ${ }_{C} O D . p d f$

[3] N. Noy, M. Sintek, S. Decker, M. Crubezy, R. Fergerson, M. Musen, Creating semantic web contents with Protégé-2000, IEEE Intelligent Systems.

[4] S. Bechhofer, I. Horrocks, C. Goble, R. Stevens, OilEd: a reason-able ontology editor for the Semantic Web, Proceedings of KI2001, Joint German/Austrian conference on Artificial Intelligence.

[5] Y. Sure, M. Erdmann, J. Angele, S. Staff, R. Studer, D. Wenke, OntoEdit: Collaborative ontology development for the Semantic Web, Proceedings of the International Semantic Web Conference (ISWC).

[6] A. Kalyanpur, B. Parsia, J. Hendler, A tool for working with web ontologies, in: In Proceedings of the International Journal on Semantic Web and Information Systems, Vol. 1, No. 1, Jan - March, 2005.

URL http://www.mindswap.org/papers/Swoop-Journal.pdf

[7] A. Farquhar, R. Fickas, J. Rice, The Ontolingua server: A tool for collaborative ontology construction, Proceedings of the 10th Banff Knowledge Acquisition for Knowledge Based System Workshop (KAW95).

[8] J. Arpírez, O. Corcho, M. Fernández-López, A. Gómez-Pérez, WebODE: a scalable ontological engineering workbench, First International Conference on Knowledge Capture (K-CAP). 
[9] J. Domingue, Tadzebao and webonto: Discussing, browsing, and editing ontologies on the web, in: 11th Knowledge Acquisition for Knowledge-Based Systems Workshop, 1998.

[10] R. J. Erich Gamma, Richard Helm, J. Vlissides, Design patterns: Elements of reusable object-oriented software, addison-wesley.

[11] S. Bechhofer, P. Lord, R. Volz, Cooking the semantic web with the owl api, Proceedings of the International Semantic Web Conference.

[12] F. Manola, E. Miller, RDF Primer W3C Recommendation. http://www.w3.org/tr/rdf-primer/.

[13] P. Patel-Schneider, P. Hayes, I. Horrocks, OWL Web Ontology Language Semantics and Abstract Syntax W3C Recommendation. http://www.w3.org/tr/2004/rec-owl-semantics-20040210/.

[14] D. Beckett, Turtle - Terse RDF Triple Language. http://www.ilrt.bris.ac.uk/discovery/2004/01/turtle/.

[15] A. Kalyanpur, C. Halaschek-Wiener, V. Kolovski, J. Hendler, Effective nl paraphrasing of ontologies on the semantic web(Technical Report).

URL http://www.mindswap.org/papers/nlpowl.pdf

[16] B. Parsia, E. Sirin, Pellet: An owl dl reasoner (poster). http://www.mindswap.org/2003/pellet.

[17] V. Haarslev, R. Möller, Description of the racer system and its applications, in: Proceedings International Workshop on Description Logics (DL-2001), Stanford, USA, 1.-3. August, 2001, pp. 131-141.

[18] I. Horrocks, The FaCT system, 1998, pp. 307-312.

URL download/1998/t98-paper.ps.gz

[19] B. Parsia, E. Sirin, A. Kalyanpur, Debugging owl ontologies, in: The 14th International World Wide Web Conference (WWW2005), Chiba, Japan, 2005, to Appear.

URL http://www.mindswap.org/papers/debuggingOWL.pdf

[20] J. Kahan, M.-R. Koivunen, E. Prud'Hommeaux, R. Swick, Annotea: An open RDF infrastructure for shared web annotations, Proc. of the WWW10 International Conference.

[21] R. Swick, E. Prud'Hommeaux, M.-R. Koivunen, J. Kahan, Annotea protocols, http://www.w3.org/2001/Annotea/User/Protocol.html.

[22] M. Klein, N. Noy, A component-based framework for ontology evolution, Workshop on Ontologies and Distributed Systems at IJCAI.

[23] L. Stojanovic, A. Maedche, B. Motik, N. Stojanovic, User-driven ontology evolution management, in: EKAW '02: Proceedings of the 13th International Conference on Knowledge Engineering and Knowledge Management. Ontologies and the Semantic Web, Springer-Verlag, London, UK, 2002, pp. 285-300. 
[24] H. L. B. Andy Seaborne, RDQL - A Query Language for RDF. http://www.w3.org/tr/owl-ref/.

[25] H. B. S. T. B. G. M. D. Ian Horrocks, Peter F. Patel-Schneider, SWRL: A Semantic Web Rule Language Combining OWL and RuleM. http://www.daml.org/2003/11/swrl/.

[26] J. Golbeck, G. Fragoso, F. Hartel, J. Hendler, P. B., J. Oberthaler, The national cancer institute's thesaurus and ontology, Journal of Web Semantics 1 (1).

URL http://www.mindswap.org/papers/WebSemantics-NCI.pdf 